\title{
Volatile Yields and Solid Grindability after Torrefaction of Various Biomass Types
}

Jean-Michel COMMANDRE, Kim LÊ THÀNH, Alexandre LEBOEUF, Patrick ROUSSET

BioWooEB Research Unit CIRAD

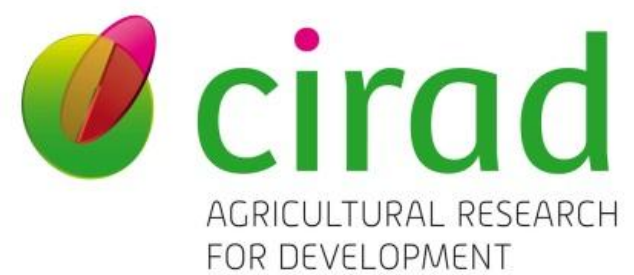




\section{The process: from biomass to fuel}

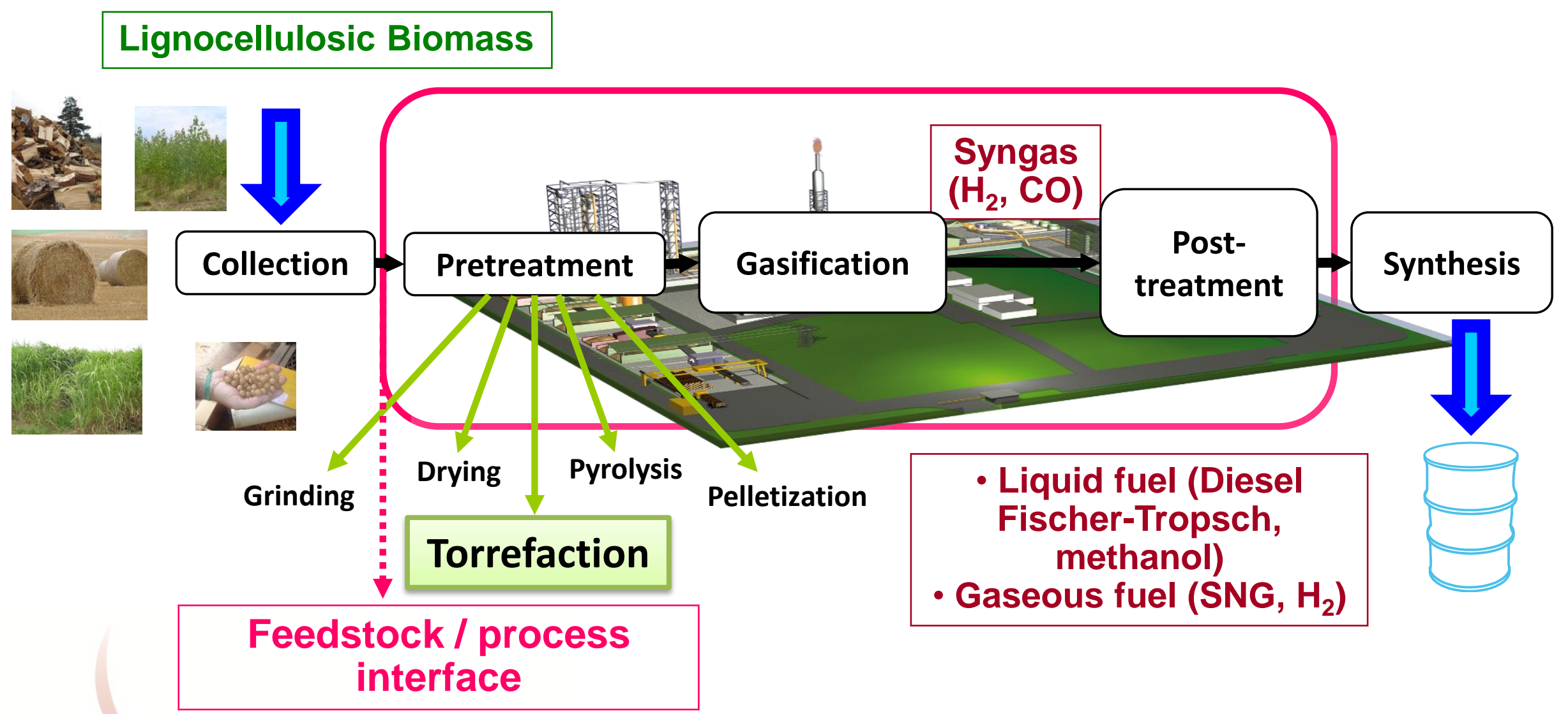

High feedstock variability $\longrightarrow$ Crucial issue for process industrialization!

$\Leftrightarrow$ Suitability feedstock / process? 


\section{Biomass torrefaction}

- Smooth thermal transformation under inert atmosphere

$\rightarrow$ Between drying and pyrolysis

$$
\left\{\begin{array}{l}
-\mathrm{T}=200-300^{\circ} \mathrm{C} \\
- \text { Residence time }=15 \mathrm{~min}-\text { several hours }
\end{array}\right.
$$

- Atmospheric pressure

\section{Biomass $\mathrm{C}_{6} \mathrm{H}_{9} \mathrm{O}_{4}$}

+ moisture $20 \%$
- Solid properties get more coal-like Suitable for entrained flow gasification
Volatile matter:

- $\operatorname{Gas}\left(\mathrm{CO}, \mathrm{CO}_{2}\right)$

- Condensable species $\left(\mathrm{H}_{2} \mathrm{O}\right.$, acids...)

Torrefied biomass

$\mathrm{C}_{6} \mathrm{H}_{8} \mathrm{O}_{3}$ +moisture $3 \%$

[ - Decrease of $\mathrm{H} / \mathrm{C}$ and $\mathrm{O} / \mathrm{C}$

- Hydrophobic nature

- Higher energy content

- Improved grindability and powder flowability 


\section{Biomasses}

Pine

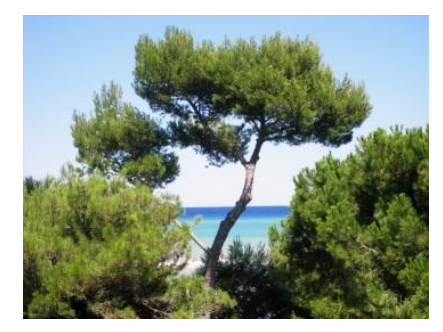

Miscanthus

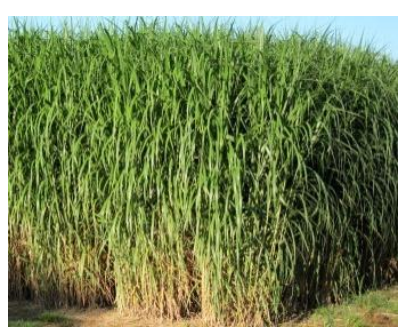

Wheat straw

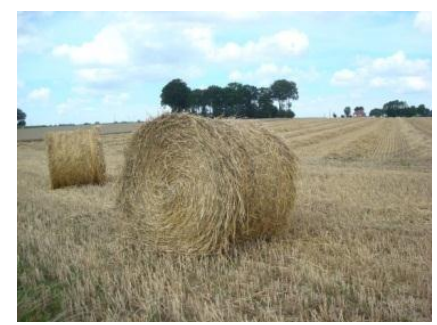

Poplar

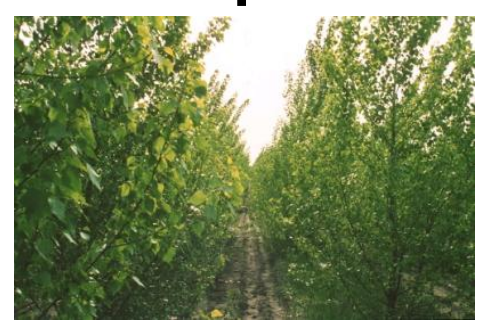

> Sampling according to XP CENT/TS 14786

$>$ Grinding $\approx \mathrm{cm}$

\begin{tabular}{|lccccccccc|c|} 
Biomass & \multicolumn{3}{c}{$\begin{array}{c}\text { Proximate analysis } \\
\text { (wt. \%) }\end{array}$} & \multicolumn{3}{c}{$\begin{array}{c}\text { Ultimate analysis } \\
\text { (wt. \% daf) }\end{array}$} & LHV \\
& Moisture & Ash (db) & VM (db) & FC (db) & N & C & H & O & (MJ/kg db) \\
\hline Pine & 11.9 & 0.3 & $85.2)$ & 14.5 & 0.2 & 49.7 & 6.1 & 44.0 & 18.1 \\
\hline Miscanthus & 8.2 & 2.2 & 80.9 & 16.9 & 0.3 & 49.2 & 6.2 & 44.3 & 18.0 \\
\hline Wheat straw & 9.0 & 6.4 & 23.5 & 20.2 & 1.0 & 49.9 & 6.1 & 43.0 & 18.4 \\
\hline Poplar & 9.0 & 3.4 & 81.0 & 15.6 & 0.2 & 51.0 & 5.9 & 42.8 & 18.4 \\
\hline
\end{tabular}




\section{Objective and working plan}

Objective:

Characterization of products released during torrefaction of various biomass nature:

$>$ Products mass balance

$>$ Solid grindability

\section{Working plan:}

> Torrefaction experiments in lab-scale reactor

$>$ Grindability tests on raw and torrefied biomasses 


\section{Lab-scale tests: \\ Products mass balance}




\section{The lab-scale device ALIGATOR}

> Samples dried at $105^{\circ} \mathrm{C}$ according to XP CEN/TS 14774
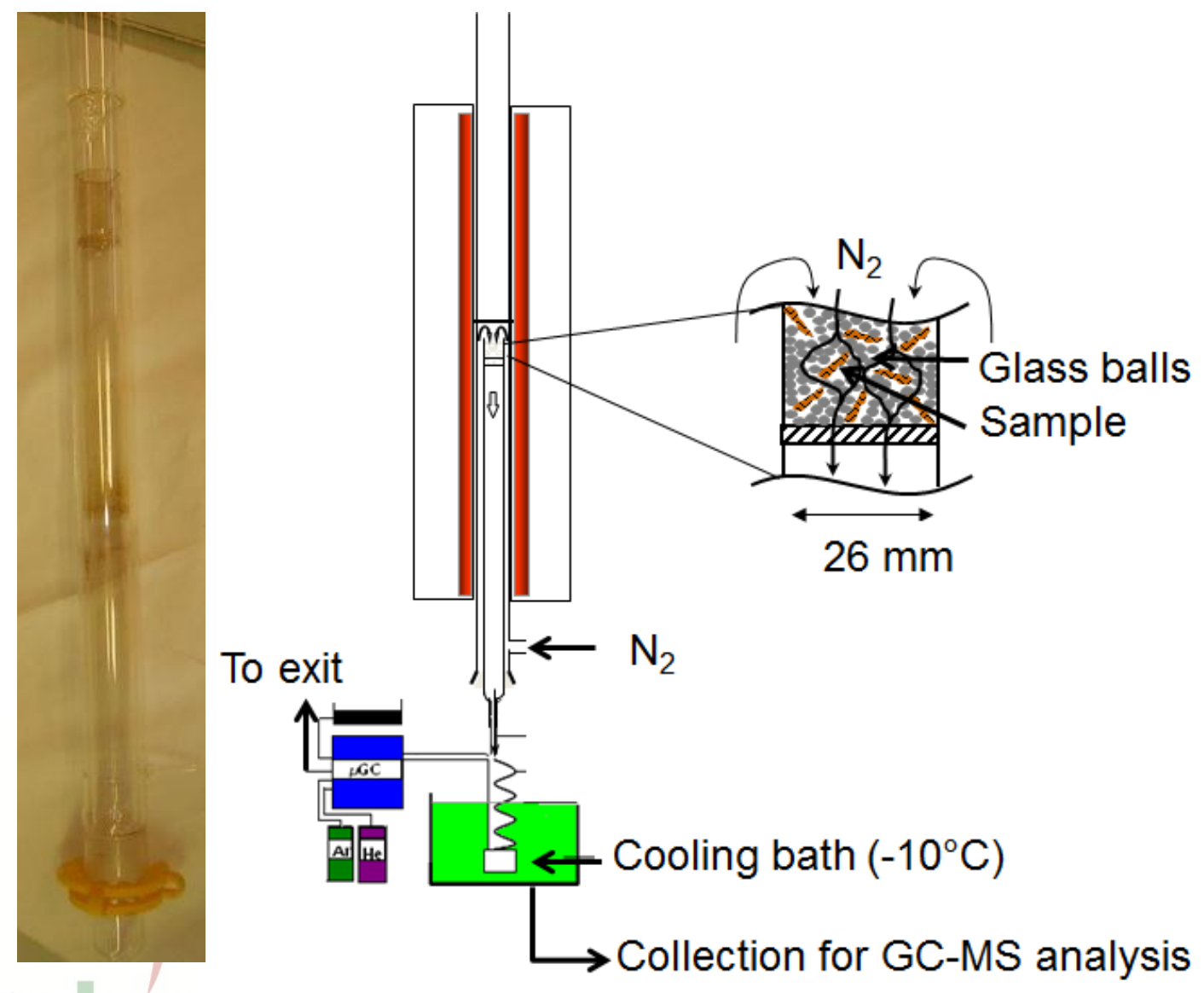

\begin{tabular}{|l|l|}
\hline Temperature & $250^{\circ} \mathrm{C}$ \\
\hline Gas atmosphere & $\mathrm{N}_{2}$ \\
\hline Gas flow & $100 \mathrm{~mL} \cdot \mathrm{min}^{-1}$ \\
\hline Pressure & atmospheric \\
\hline Sample mass & $\sim 1.5 \mathrm{~g}$ \\
\hline
\end{tabular}

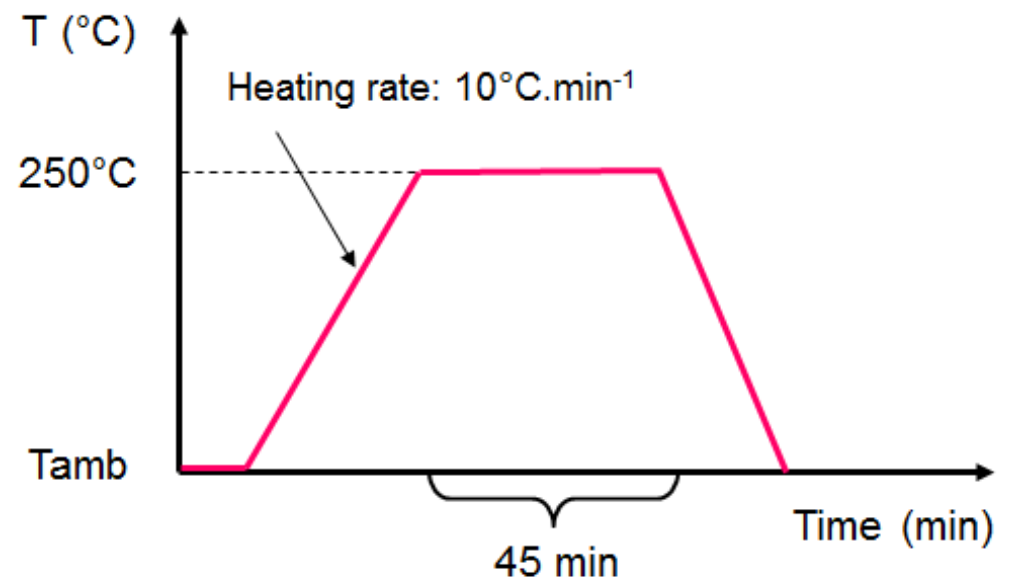




\section{Global mass balance}

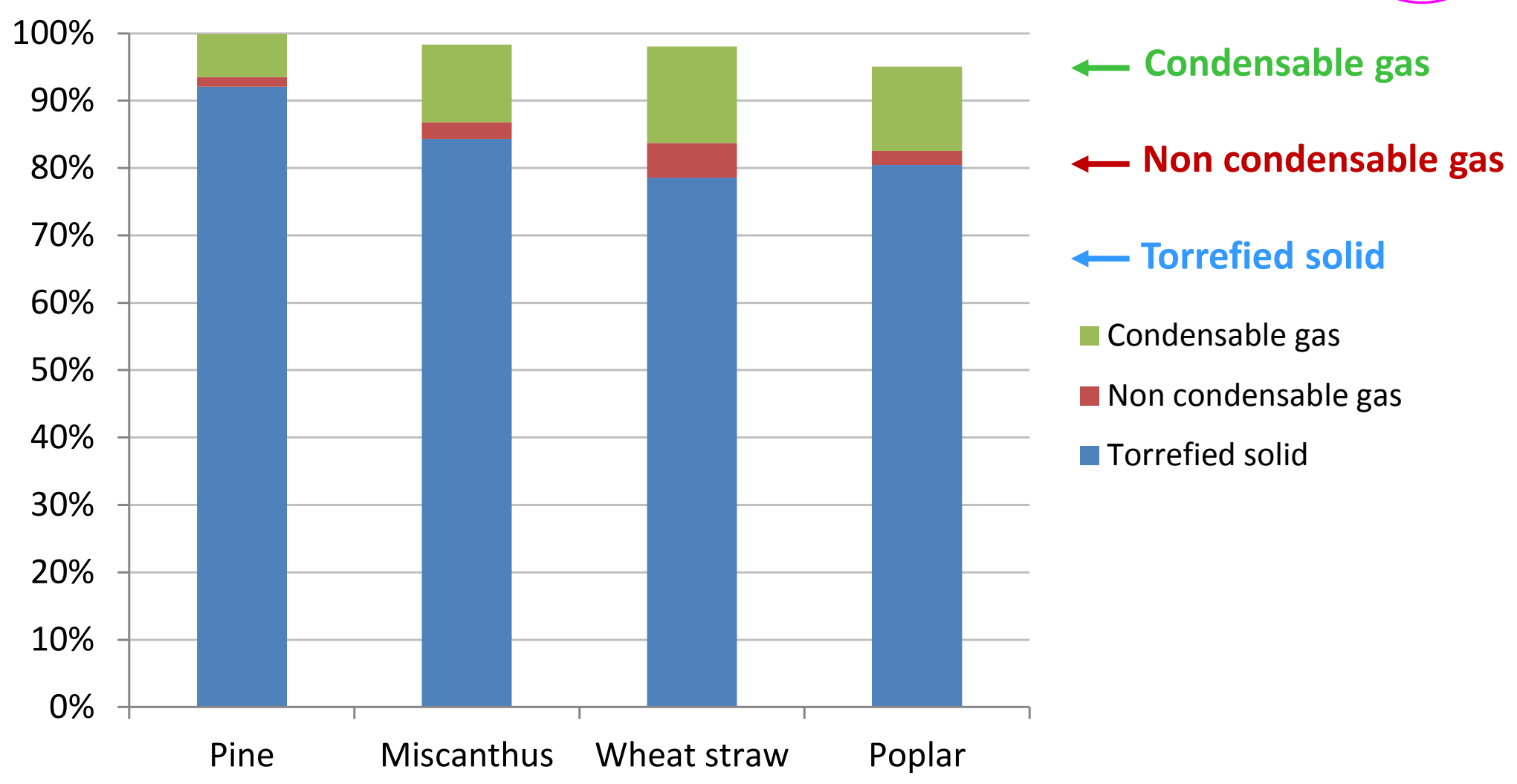

- Global distribution depends on nature of biomass

$\stackrel{H}{\Rightarrow}$ Higher mass loss for agricultural by-products and SRC

- For all biomass types: Volatile species are mainly condensable 


\section{Mass balance: Volatile species}

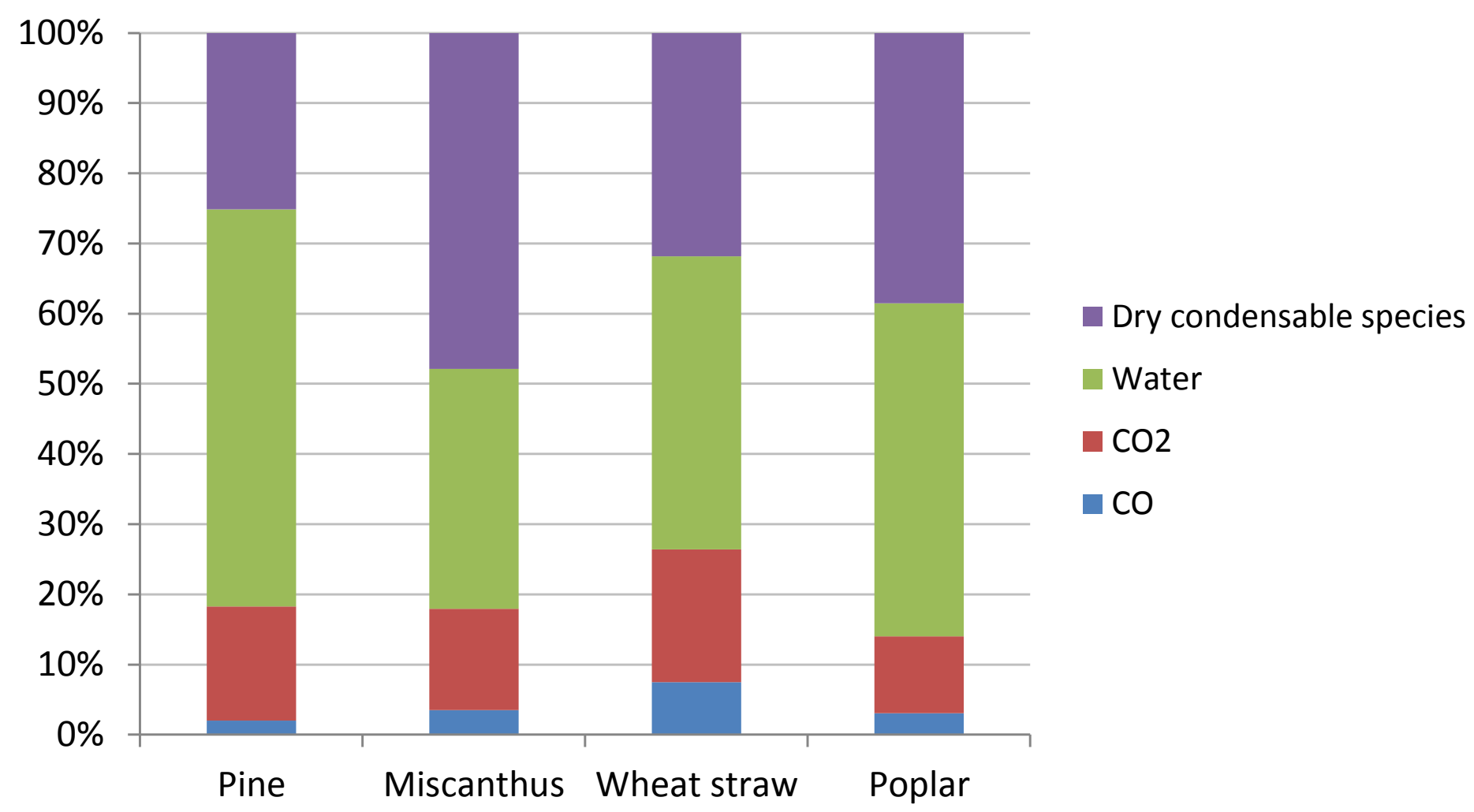

- Water and dry condensable: difference softwood/agricultural biomass

- CO: high content for agricultural by-products

$\stackrel{\leftrightarrow}{\rightarrow}$ Litt: $\mathrm{CO}_{2}<=$ decarboxylation of acid groups

$\mathrm{CO}$ ? Not clearly explained; catalytic reaction between $\mathrm{CO} 2$ and $\mathrm{C}$ ? 


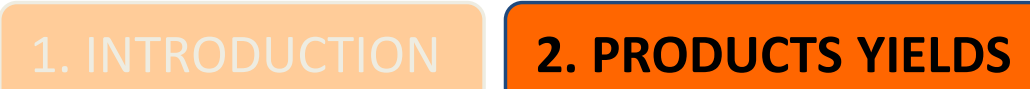 \\ Mass balance: Volatile species}

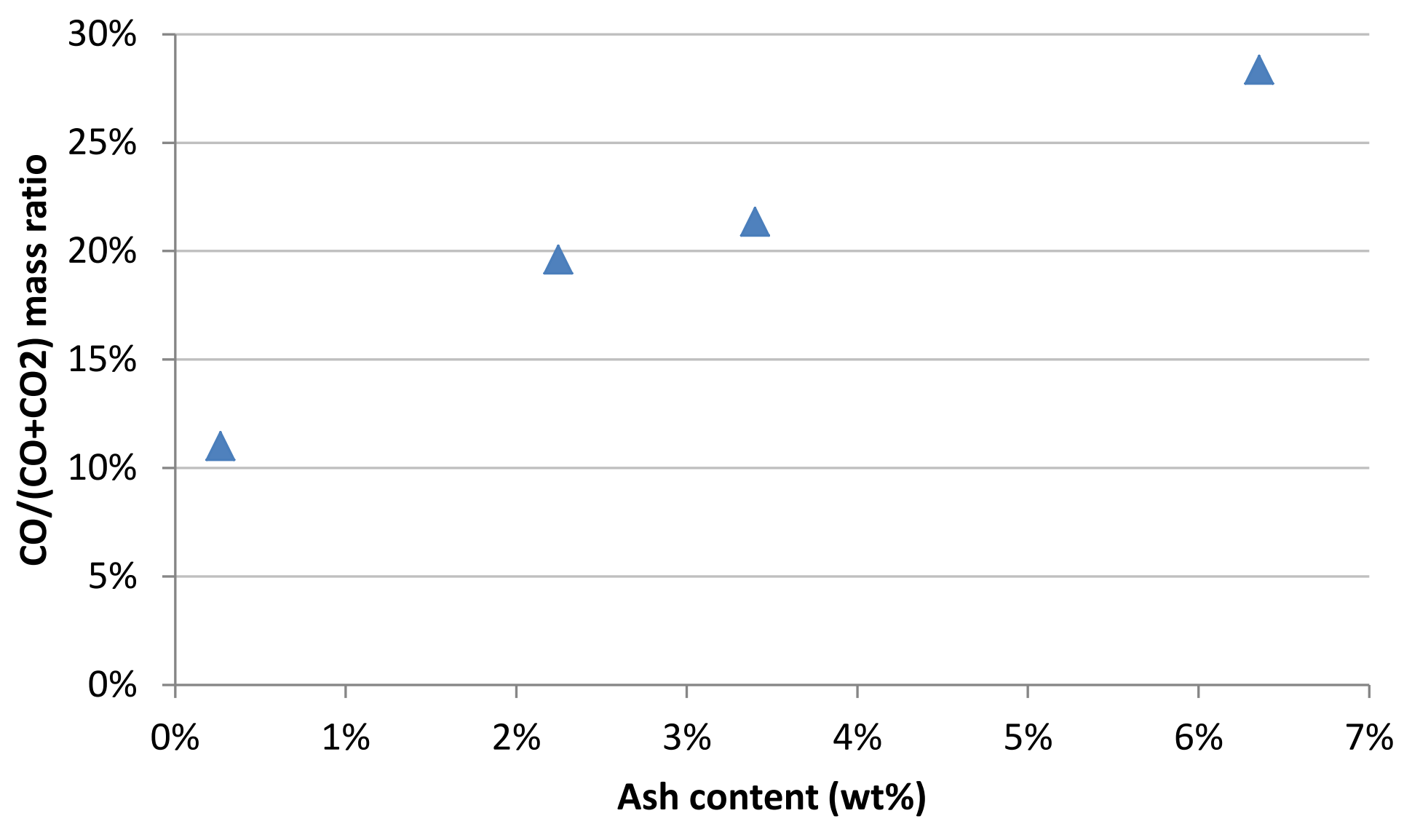

- $\mathrm{CO} / g a s$ ratio increases with ash content

$\stackrel{\leftrightarrow}{\rightarrow}$ Mineral matter catalytic effect 


\section{Mass balance: dry condensable species}

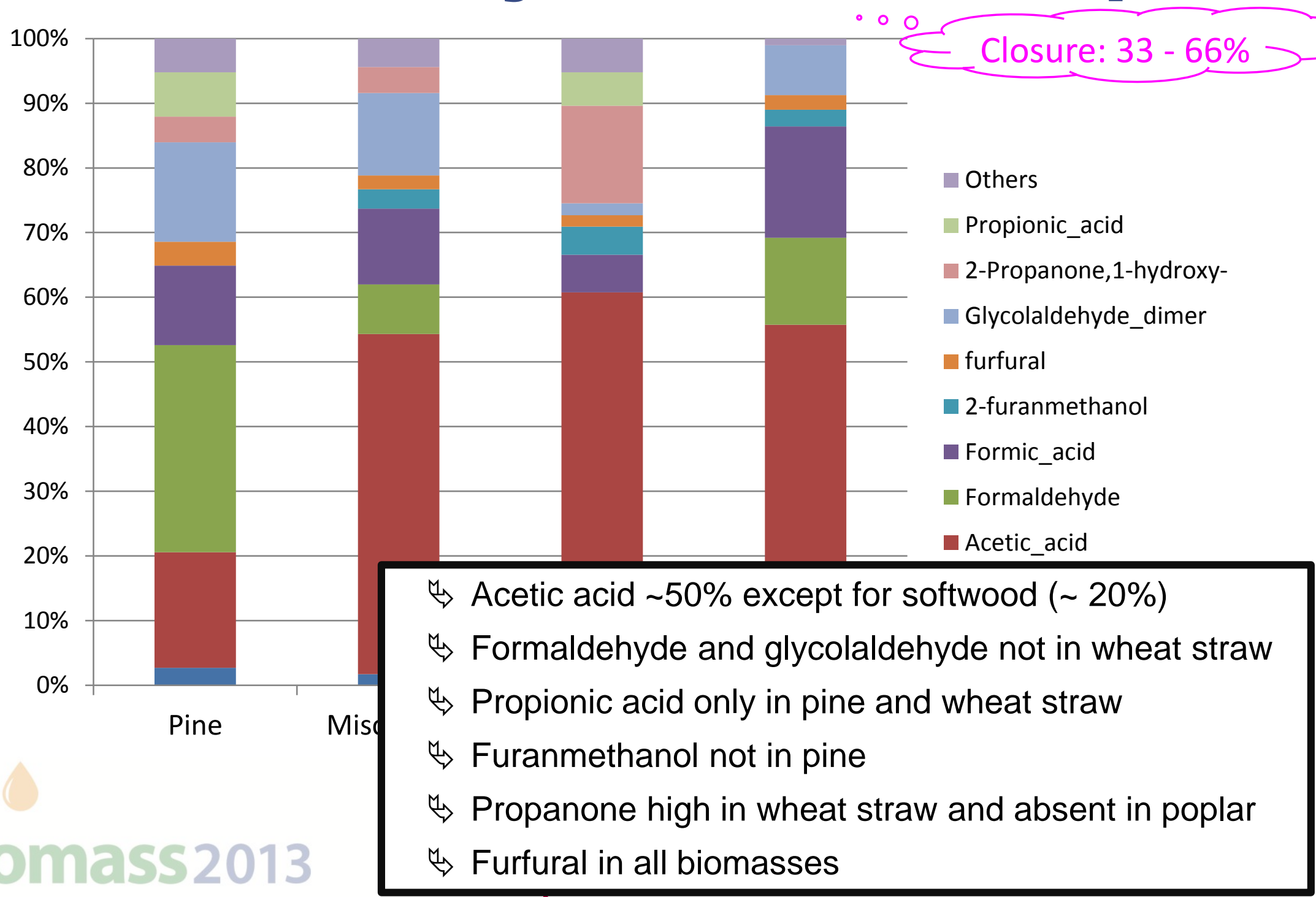




\section{Torrefied solid composition}

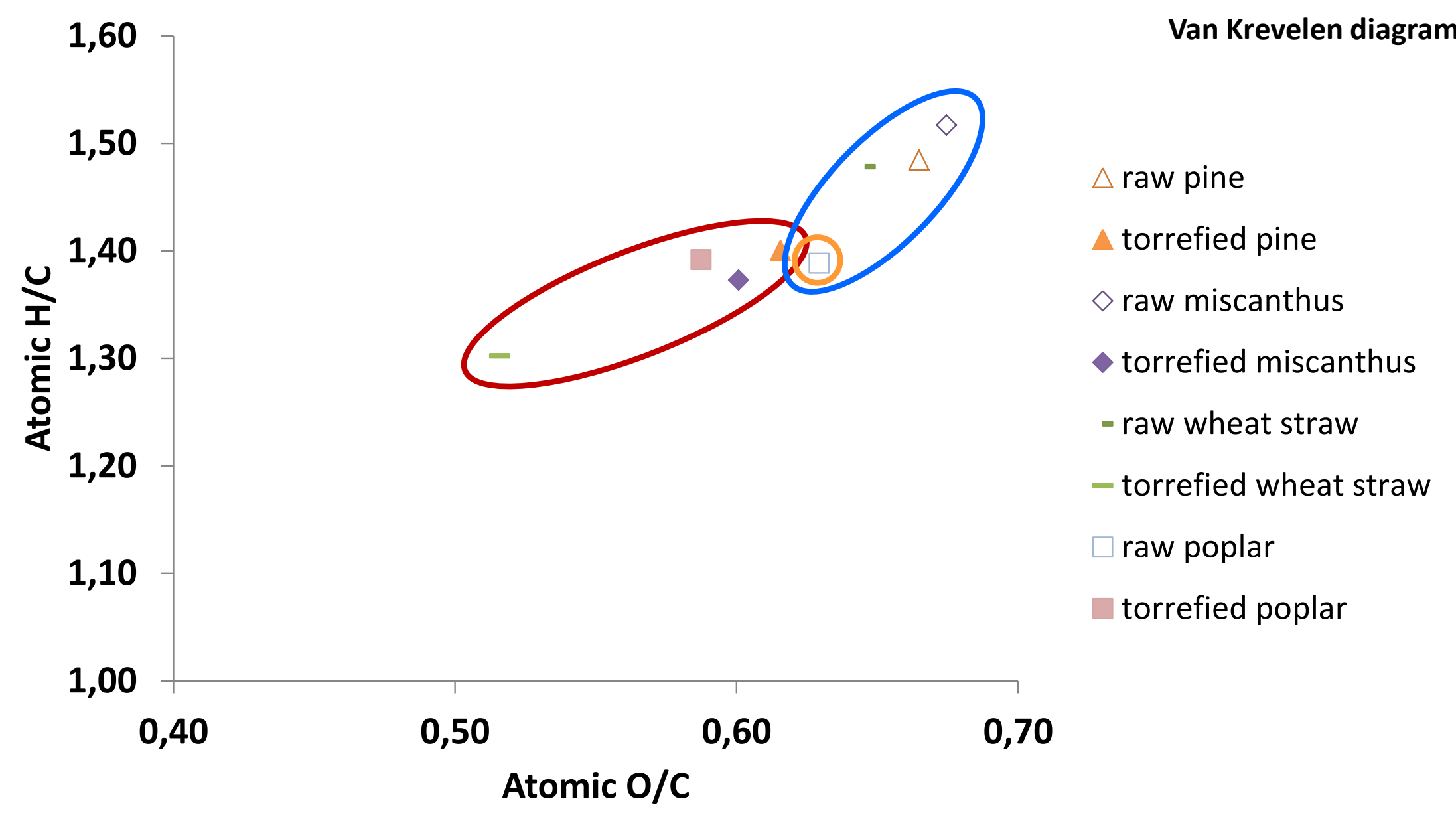

- Ratios $\mathrm{H} / \mathrm{C}$ and $\mathrm{O} / \mathrm{C}$ are similar for all raw biomasses, except for poplar 


\section{Torrefied solid: energy yield}

$$
\text { Energy yield (\%) }=\frac{\mathrm{M}_{f}}{\mathrm{M}_{o}} \frac{\mathrm{GCV}_{f}}{\mathrm{GCV}_{o}} \times 100 \quad \text { (Arias, 2008) }
$$

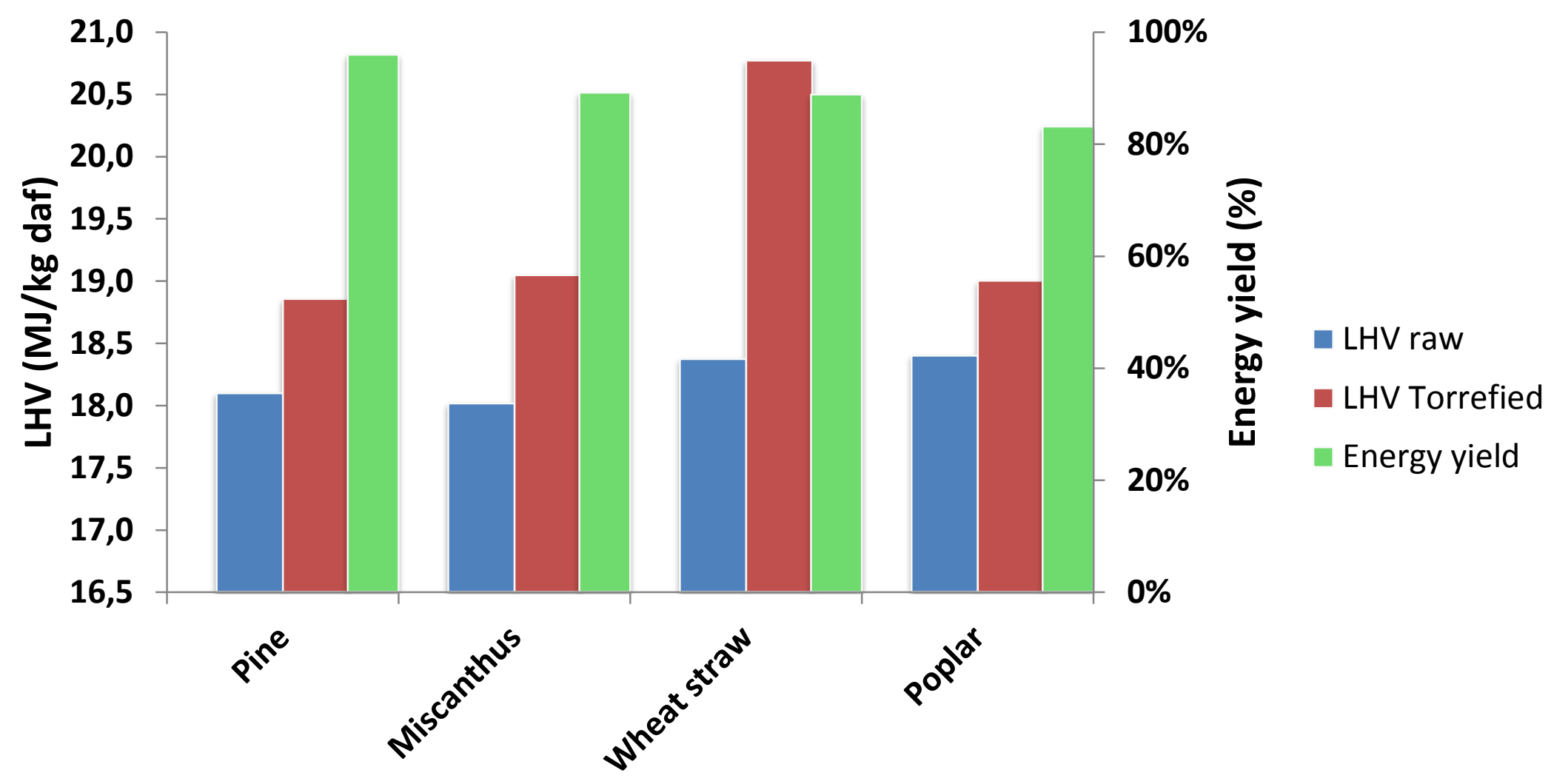

LHV similar for raw biomasses ; LHV increases after torrefaction

- Highest energy yield for pine 


\section{Grindability tests}




\section{Grinding device}

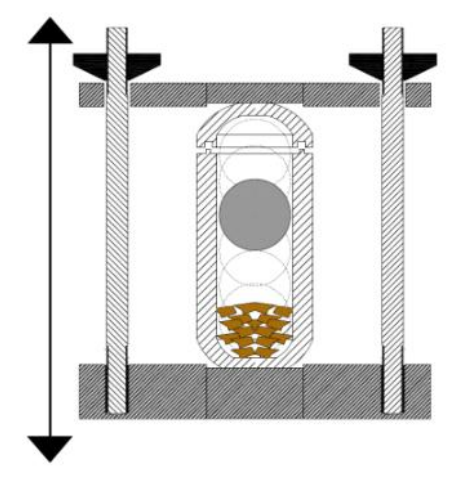

- Ball mill

- Protocol

$\stackrel{4}{4}$ volume of biomass: $50 \mathrm{~cm}^{3}$

$\Leftrightarrow$ grinding time: 1 min

\section{Particle size distribution}

- Sieving following standard NF EN 15149

- Low weight sieves of $\phi=60 \mathrm{~mm}$

$\Leftrightarrow 6$ sieves from $1 \mathrm{~mm}$ to $50 \mu \mathrm{m}$

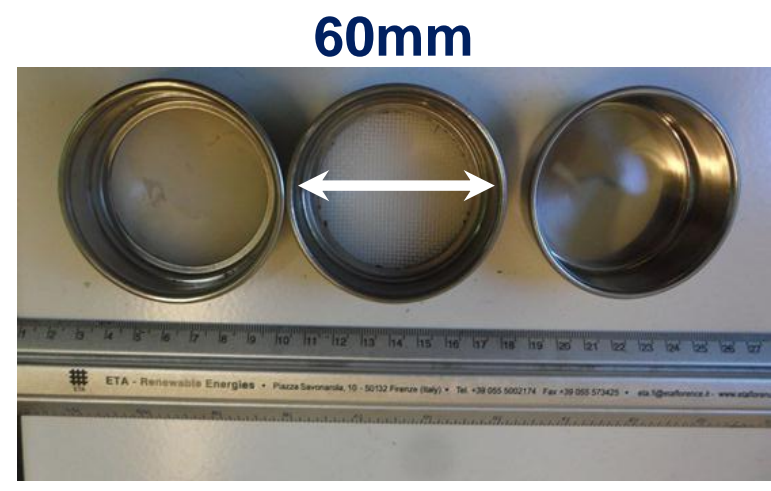




\section{Torrefaction protocol}

Objective:

To assess influence of nature of biomass on grindability

$\Rightarrow$ mass loss 17\% for all biomasses

$\Leftrightarrow$ Adjustment of torrefaction temperature

\begin{tabular}{lcc}
\hline & $\begin{array}{c}\text { Torrefaction } \\
\text { temp. }\left({ }^{\circ} \mathbf{C}\right)\end{array}$ & $\begin{array}{c}\text { Mass loss } \\
\text { (wt. \%) }\end{array}$ \\
\hline Pine & 275 & 17.0 \\
Miscanthus & 250 & 16.9 \\
Wheat straw & 240 & 17.2 \\
Poplar & 245 & 17.6 \\
\hline
\end{tabular}

\section{Relative grinding energy}

Assumptions:

* Proportional to surface created

* Particles are spherical

* Same density whatever particle size

$\Longleftrightarrow$ Relative grinding energy $=\frac{1}{\text { Cumulative mass fraction }}$ 


\section{Particle size distribution}

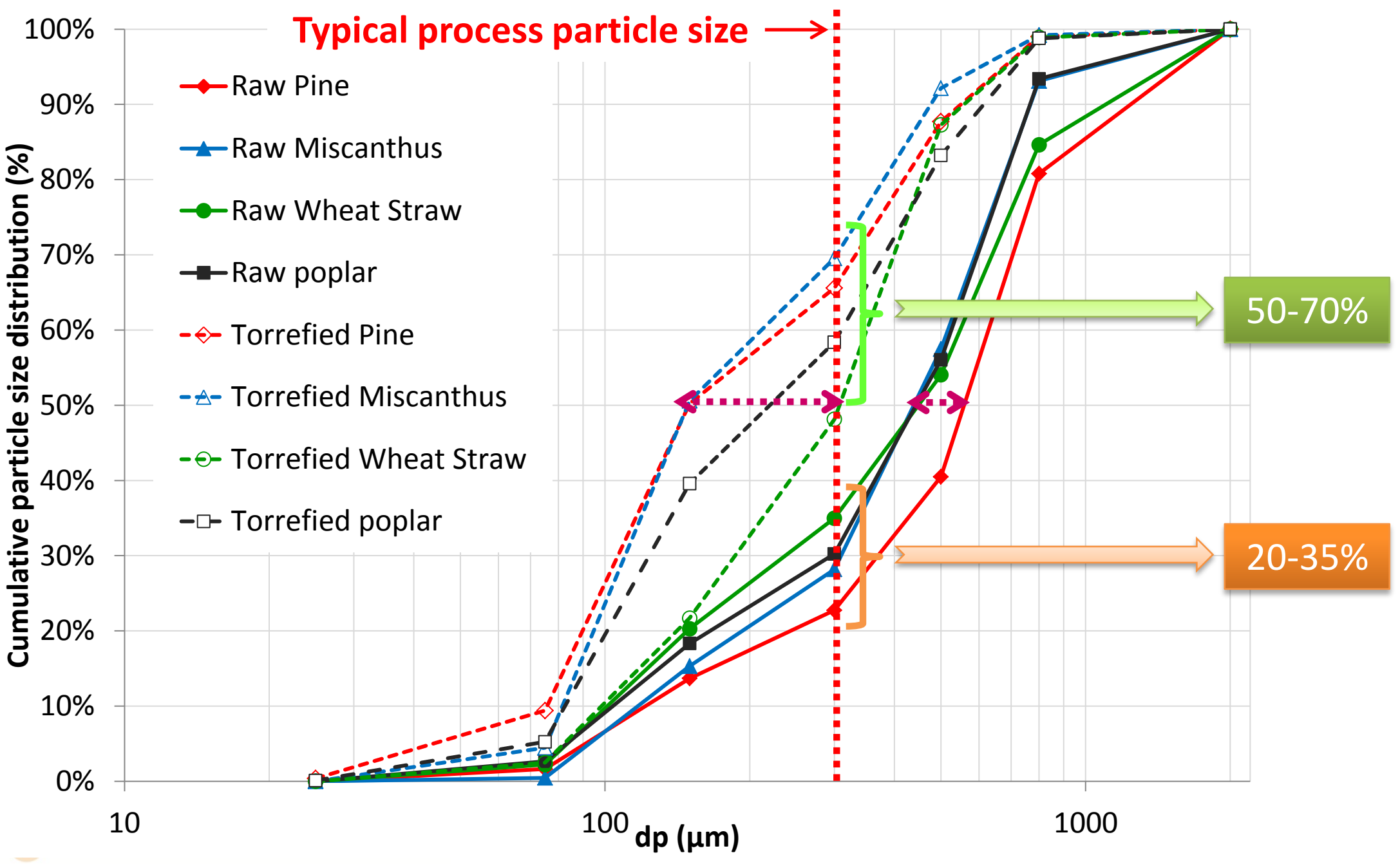

- Smaller particle sizes after torrefaction than raw biomass

- Large fraction of torrefied particles suitable to gasification 


\section{Relative grinding energy}

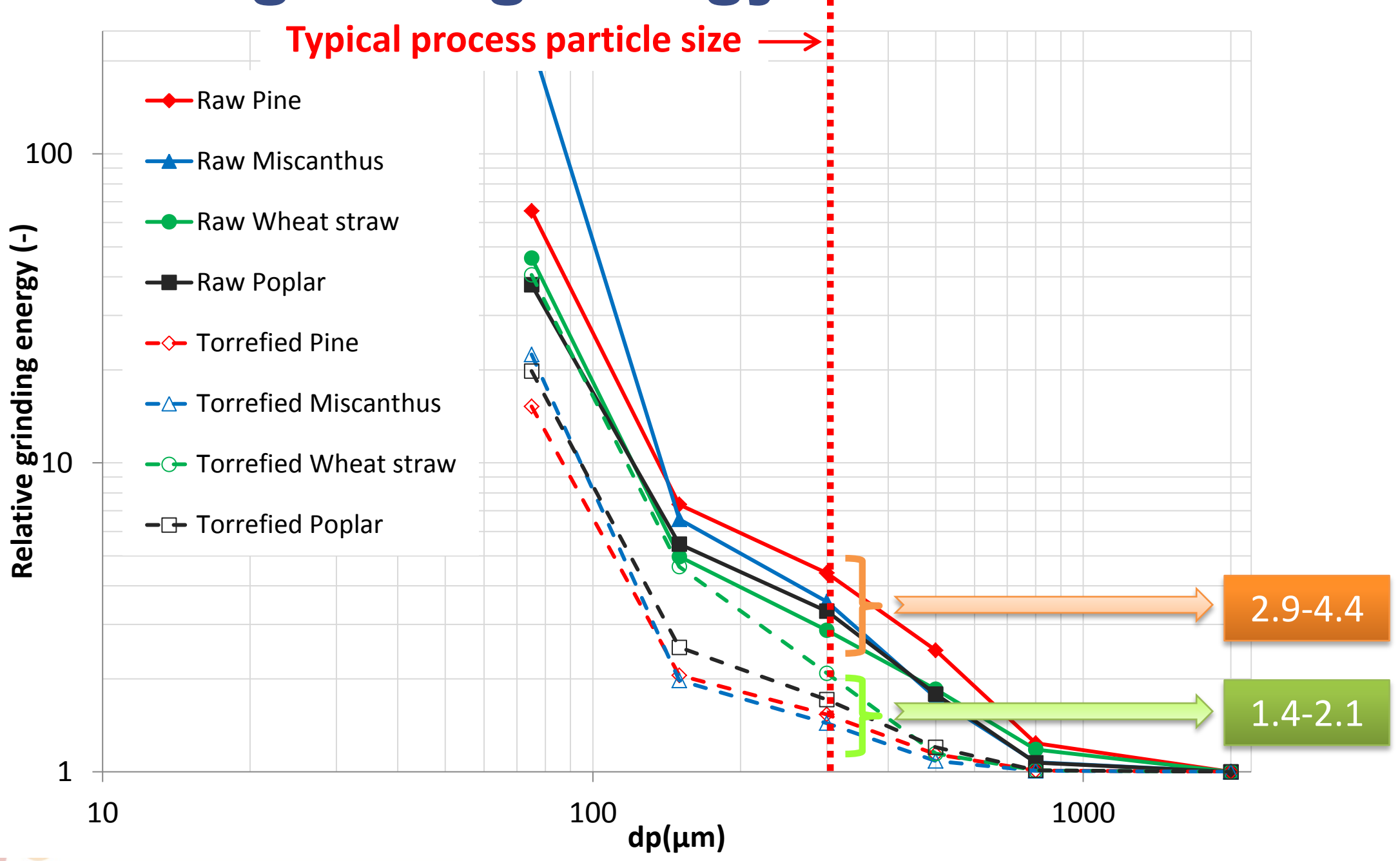

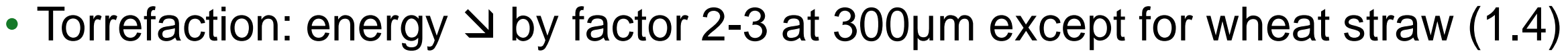




\section{Conclusion}

Properties of products released during torrefaction of various biomasses?

- Torrefaction improves biomass grindability:

$>$ Grinding energy significantly reduced by torrefaction

$>$ Trend less marked for wheat straw

(-) Pine, Miscanthus, SRC

\section{Wheat straw}

- Gaseous products composition depends on nature of biomass

$>$ To be considered for cleaning step

$>$ Interesting for species valorization in biorefinery process

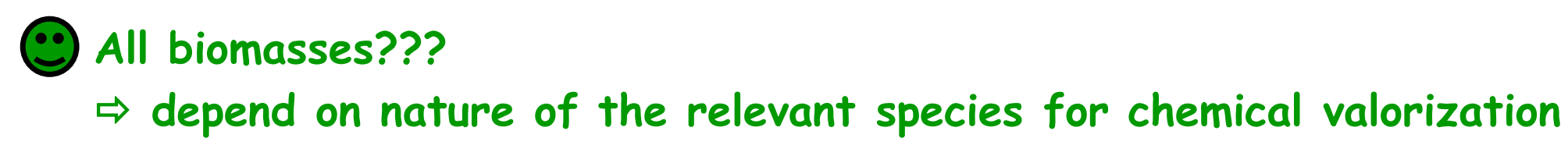

(-) All biomasses???

$\Rightarrow$ depend on nature of the relevant species for chemical valorization

\section{What's next?}

- Tests on other samples

- Pilot scale tests $\Rightarrow$ continuous torrefaction

- Comparison of grindability tests with measures on grinding energy at large scale

- Improvement of condensable species quantification 
